\title{
Heavy and Light Monopoles in Magnetic Reversion in Artificial Spin Ice
}

\author{
Alejandro León \\ Facultad de Ingeniería, Universidad Diego Portales, Santiago Chile
}

\begin{abstract}
This work makes a theoretical study of the dynamics of emergent elemental excitations in artificial spin ice systems with hexagonal geometry during the magnetic reversion of the system. The magnetic and physical parameters of the nanoislands that form the array are considered as variables in the study. The parameters considered are: the energy barrier for the inversion of each nanoisland, the magnetic moment of the nanomagnets and the possible disorder in the sample. Our results show that the reversion dynamic presents two distinct mechanisms of magnetic reversion, with different elemental excitations for each mechanism. The first mechanism presents a reversion with the appearance of magnetic monopoles that do not move in the samples (heavy monopoles) and the absence of Dirac chains. In the other mechanism elemental magnetic excitations (light monopoles) appear that move great distances in the sample, giving rise to extensive Dirac chains during the magnetic reversion.
\end{abstract}




\section{Introduction}

Artificial spin ice systems have been intensely studied theoretically and experimentally in recent years [1-9] because they present magnetic charge defects with similar characteristics to the magnetic monopoles postulated by Dirac [10]. The main advantage of these systems lies in the fact that studies can be made of the dynamic of these elemental excitations at ambient temperature because the nanoislands have energy reversion barriers above the thermal fluctuations at this temperature. The work of Ladak et al. [7] showed the direct observation of these elemental excitations in a cobalt nanoisland array with hexagonal geometry. Another recent study [8] showed the experimental evidence of magnetic reversion through Dirac chains in an artificial spin ice system with hexagonal geometry. The work of Mengotti et al. [8] includes the statistics that demonstrate the decrease in the dimensionality of the system based on the propagation of Dirac chains, with mobile elemental excitations (monopoles) at their ends. Another recent experimental work [9] verified that magnetic reversion is produced independently in a square network for the two sub-networks that form the array. As well, the same study verified that the reversion is produced through unidimensional cascades of the parallel nanomagnets of the system. Some studies have been published on the effect of the size of the system in artificial spin ice arrays on energy minimization protocols [11] and the effect of the edges on vertice dynamics [12]. A recent study [13] was published on the effects of the size of finite systems, the aspect ratio of the system and the concentration of impurities on the dynamic of emergent monopoles in a hexagonal network during magnetic reversion. This work establishes that the density of mobile monopoles depends on the size, concentration of impurities and shape of the bidimensional system.

The current techniques of synthesizing artificial spin ice systems [1, 7-9] allows for controlling the size and shape of the system and the magnetic properties of the individual nanoislands. However, there is still no fine control of the relationship between the magnetic moments of each nanoisland and the external magnetic field necessary to provoke magnetic reversion in isolated nanoislands, giving rise to the possibility of having a broad spectrum of combinations of these two variables. In this work we studied the dynamic of magnetic reversion in a hexagonal array of nanomagnets in function of the magnetic properties of the individual components of the array. Our results show that there are two different reversion mechanisms with two distinct types of emergent magnetic monopoles, depending on the 
energy barrier for the reversion of the nanomagnets and on their magnetic moment. In one case monopoles emerge and remain in repose during the reversion. We term this type of excitation "heavy monopoles". In the other case, elemental excitations emerge that move great distances through the sample generating extensive Dirac chains. We term this type of excitation "light monopoles".

\section{Emergent Magnetic Monopoles}

The system that we studied is a bidimensional array of magnetic nanoislands on a hexagonal network. Three nanomagnets converge in each vertice of the array (except on the edges). We assign a magnetic load value to each vertice of the array in function of the magnetic poles present in the vertice. The north pole of each nanoisland is associated with a $q=+1$ charge and the south pole is associated with a $q=-1$ charge. This implies that each vertice of the array has the following net charge values: $Q_{1}=+1, Q_{2}=-1, Q_{3}=+3$ and $Q_{3}=-3$. If the system is submitted to a magnetic field along the $x$-axis, as shown in the upper part of Figure 1, the vertices in the array (except at the edges) acquire a charge of +1 or -1 . If the samples are initially magnetized, to the left, for example, and we apply a magnetic field in the inverse sense, elemental excitations appear (magnetic monopoles) in the vertice. The monopoles are defined as in reference [8]. When the samples are totally magnetized to the left, a positive monopole emerges if the net charge of the vertice "A" goes from $Q=-1 \rightarrow Q^{\prime}=+1 \Rightarrow \triangle Q=+2$. Equally, a negative monopole results if vertice "A" goes from $Q=-1 \rightarrow Q^{\prime}=-3 \Rightarrow \triangle Q=-2$. In the case of the type B vertices, a negative monopole emerges if $Q=+1 \rightarrow Q^{\prime}=-1 \Rightarrow \triangle Q=-2$ and a positive monopole emerges when $Q=+1 \rightarrow Q^{\prime}=+3 \Rightarrow \triangle Q=+2$. The monopoles emerge in pairs and then move away from each other but connected through Dirac chains, as a product of the applied magnetic field, as can be observed in the lower part of figure 1. A possible combination could occur if $\triangle Q= \pm 4$, but these events have not been observed in experiments or simulations. Of the emergent monopoles only those that maintain at least one of the nanomagnets that converge in the vertice are mobile, with their component over the $x$-axis directed to the left. We termed the total quantity of mobile monopoles for each site of the network the "density of mobile monopoles" with the designation $\sigma_{M}$. When the conditions $\triangle Q= \pm 2$, are present in a vertice, but with all the nanomagnets, that converge in the vertice, with the component 
of the magnetic moment directed to the right, monopoles emerge that are termed "trapped monopoles" [8] and in this work are not considered as monopoles properly but rather as "charge defects". We termed the quantity of these defects per site of the hexagonal network as "defect density" and denote it by $\rho$.

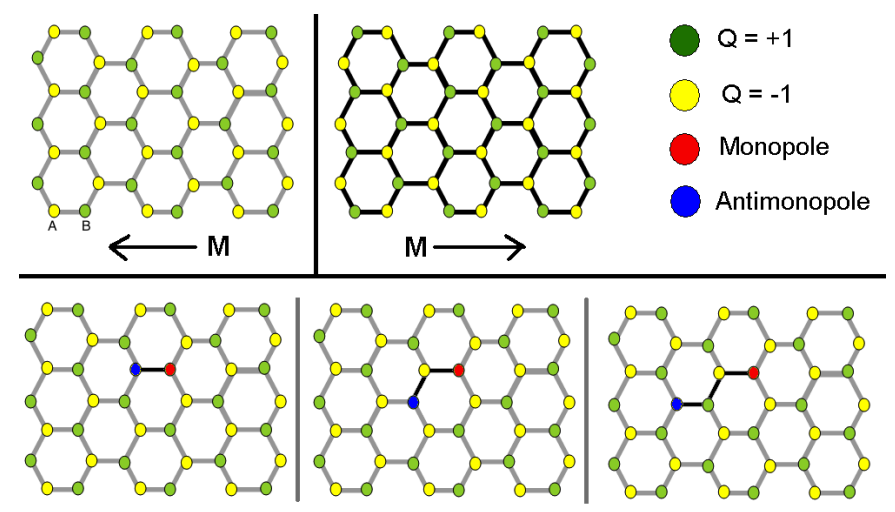

Figure 1. The upper left part of the figure shows the array of nanoislands magnetized to the left and the upper middle part of the figure shows the array of nanoislands magnetized to the right. All the nanoislands that have a negative value for the $x$ component of the magnetic component are represented by gray lines. In the case that the $x$ component of the magnetic moment of the nanoisland is positive, it is represented by a black line. The upper right part of the figure shows the color code for the vertices and the emergent monopoles.

\section{The studied system and the model used}

The system studied, is a sample of $(23 \mu m \times 23 \mu m)$ and 1,144 nanomagnets, with impurities. The lattice constant (the distance between two adjacent vertices) has a value of $a=577 \mathrm{~nm}$. The random magnetic moment of individual islands is given by $m=m_{0} \beta$, where $\beta$ is a dimensionless Gaussian random variable with $\langle\beta\rangle=1$ and $s \equiv\left(\left\langle\left(\beta-\langle\beta\rangle^{2}\right)\right\rangle\right)^{1 / 2}$. We studied the dynamic of emergent monopoles during magnetic reversion in function of the $m_{0}$ moment of the individual nanoislands; of $H_{b}$, the necessary field to revert the moment of the individual nanoislands and of $s$, the concentration of impurities in the sample. Using a model based on a frustrated cellular automata (FCA), we consider the dipolar interaction between the magnetic moments in the Hamiltonian of the system and the dynamic evolution of the system. The mathematical basis of the model and its applications are explained in detail in reference [14]. Likewise, the results of reference [13] validate our model using experimental 
data from the work of Mengotti et al. [8]. In the FCA, the automaton is updated in several steps (three in this case), minimizing the total energy total of the system, evaluated with dipolar interaction.

\section{Heavy and light monopoles}

The methodology used in our work consists of fixing the magnetic field $H_{b}$, necessary to revert an isolated nanoisland and simulate magnetic reversions of the array for different values of $m_{0}$. Just one simulation provided us with information of the hysteresis curve, the total density of impurities $\rho$ and the density of mobile monopoles in function of the applied magnetic field. During the magnetic reversion of the system, the quantity of mobile monopoles increases, reaches a maximum, for a field value close to the coercivity field and then decreases to zero. As relevant data of the simulation, we use the maximum value reached of the density of the $\sigma_{M}$ mobile monopoles. We term this maximum value $\sigma_{\max }$. Because we simulated the presence of impurities in the system, with a random procedure we repeated the simulation for each experiment 100 times and determined $\left\langle\sigma_{\max }\right\rangle$ and the standard error of the measured samples. Figure 2 shows the results for four values of the reversion field of the nanoislands. Each graph shows the maximum value of the average density of the mobile monopoles in function of the magnetic moment $m_{0}$ of the nanoislands. In each case, three concentrations of impurities are considered. We can appreciate two distinct behaviors in all the graphs. A first region, dominated by the presence of heavy monopoles, where the magnetic reversion occurs with the appearance of elemental excitations, but these do not move in the sample and remain static. In this region, the samples with few impurities present a higher number of monopoles, reaching $100 \%$ in 3 situations shown in Figure 2. This is explained by the fact that the disorder in the system allows that diagonal nanoislands join two horizontal nanoislands that have heavy monopoles in their extremes and thus the number of these monopoles decreases without the movement of these excitations. This situation is shown in Figure 4.1. When the system has a low percentage of impurities, the probabilities of these unions is very low and consequently all the sites of the network end up with a heavy monopole. Similarly, we can note in Figure 2 that there is a threshold value for the magnetic moment $m_{0}$. This threshold value separates the region of heavy monopoles from the region of light monopoles. Monopoles are generated in the light excitation region that 
can move large distances in the system, extending the Dirac chains that join the monopole pair created, which is presented in Figure 4.3. The impurities in this region play a distinct role from the previous case, given that it permits the appearance of more monopole pairs and consequently increases $\sigma_{\max }$. density. The upper right of Figure 2 shows the results with the experimental parameters used in validating the model [13], with $m_{0}=1.1 \times 10^{-15} \mathrm{~A} \cdot \mathrm{m}^{2}$ the experimental value for nanoisland moment.

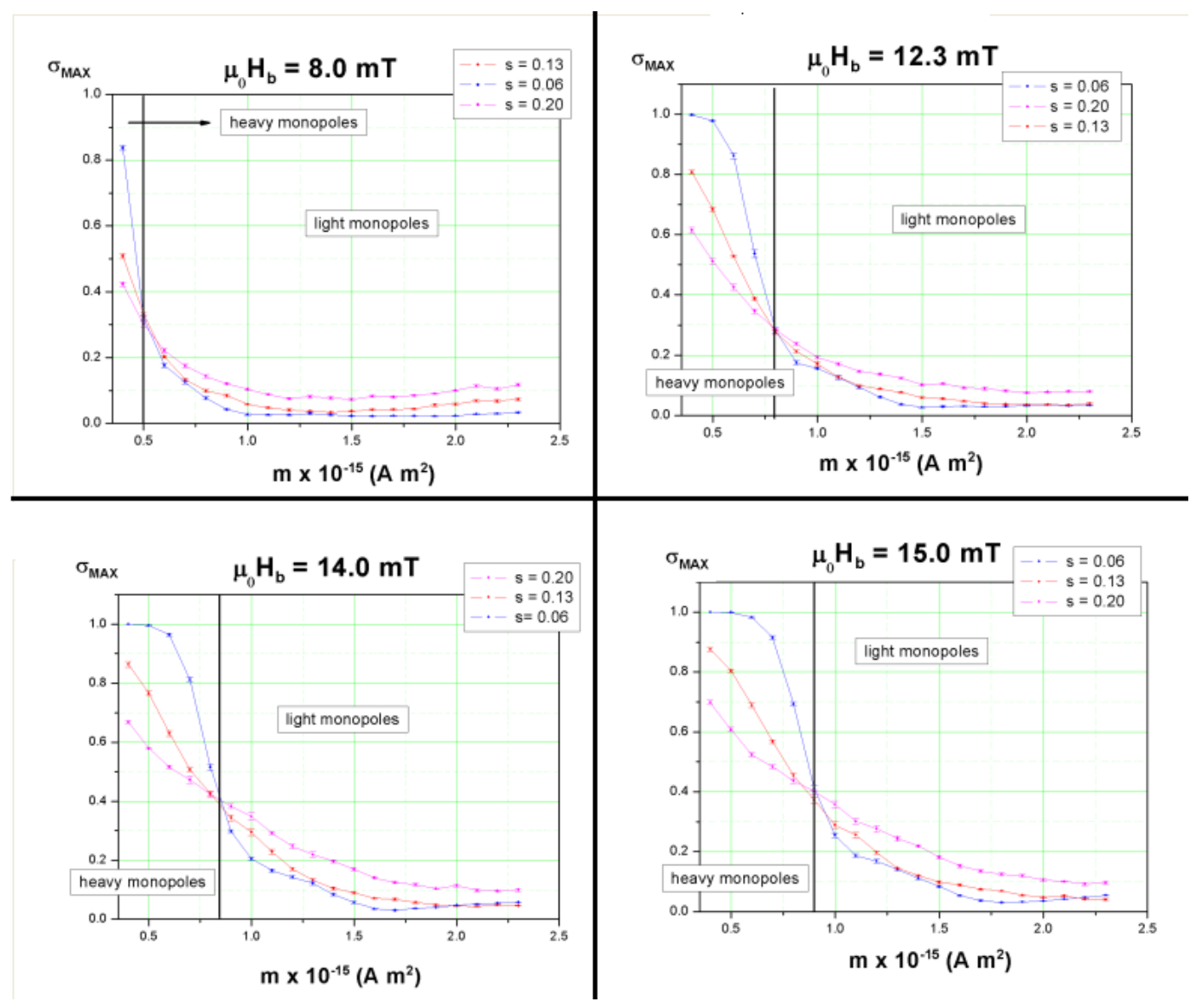

Figure 2. Maximum density of mobile monopoles in function of the magnetic moment of the nanoislands that form the array. The graphs represent four field values to revert the magnetic moment of the nanoislands.

Figures 3 and 4 show simulations with these experimental parameters, except for $m_{0}$. Figure 4.1 shows the situation in the case of heavy monopoles $m_{0}=0.4 \times 10^{-15} \mathrm{~A} \cdot \mathrm{m}^{2}$, Figure 4.2 shows the reversion in the threshold value of the magnetic moment of the nanoislands $m_{0}=0.8 \times 10^{-15} A \cdot m^{2}$ and Figure 4.3 shows reversion in the light monopoles regime $m_{0}=1.5 \times 10^{-15} A \cdot m^{2}$. We can note in Figure 4.2 that the magnetic reversion in the threshold value is produced with the coexistence of heavy and light monopoles. 

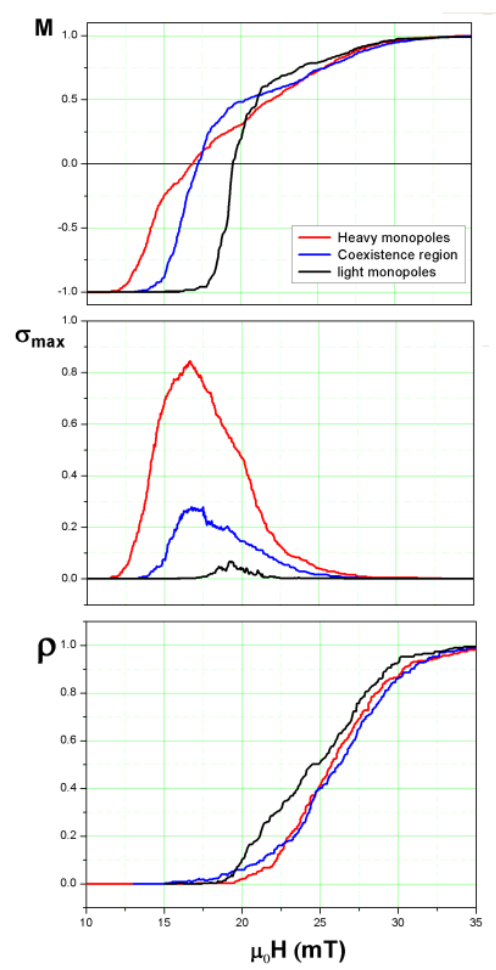

Figure 3. Magnetization $M$, maximum density of $\sigma_{\max }$ monopoles and the density of defects $\rho$ for the three simulations shown in Figure 4. The red curves correspond to the simulation shown in

Figure 4.1, the blue curves correspond to Figure 4.2 and the black to Figure 4.3.

Figure 3 shows the hysteresis curve, the maximum density of monopoles and the density of defects for the simulations, which is shown in Figure 4. We can note that coercivity is reached in the heavy monopole regime before it is in the light monopole regime. In the light monopole regime magnetization goes abruptly from a value of -1 al to 0.5 . It is interesting to note that this characteristic could be desirable to use this type of system to propagate binary information [15]. A species of plateau is then produced for the three curves. 


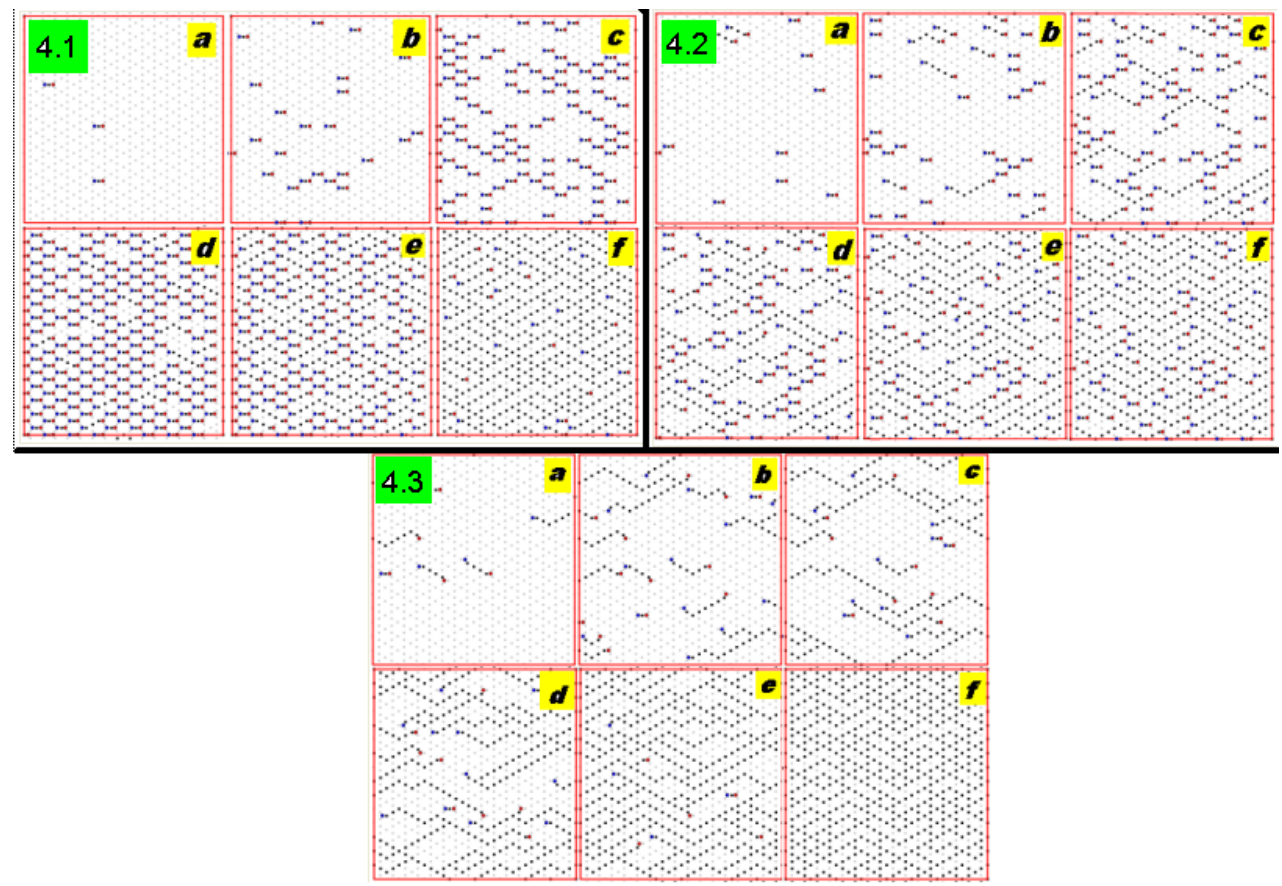

Figure 4. Simulation of the magnetic reversion for the parameters $\mu_{0} H_{b}=12.3 m T, s=0,13$, of the experimental work [8], except for the magnetic moment. Figure 4.1 shows a simulation in the heavy monopole regime, $m_{0}=0,4 \times 10^{-15} \mathrm{~A} \cdot \mathrm{m}^{2}$, Figure 4.2 shows the region of coexistence of heavy and light monopoles, $m_{0}=0,8 \times 10^{-15} \mathrm{~A} \cdot \mathrm{m}^{2}$ and Figure 4.3 shows light monopoles regime, $m_{0}=1,5 \times 10^{-15} A \cdot m^{2}$. Each simulation shows the central region of the nanoisland array during the magnetic reversion for six external magnetic field values. The gray squares represent nanoislands with the $x$ component of the magnetic moment directed to the left and the black squares to the right. The red circles represent positive monopoles and the blue circles negative monopoles. In the three situations, figures $\mathrm{a}, \mathrm{b}$ and $\mathrm{c}$ correspond to values of the external magnetic field, lower than the coercivity field $H<H_{C}$. Figure d represents the case for $H=H_{C}$ and figures e and f represent situations in which $H>H_{C}$.

In our results, we can observe that the length of the Dirac chains present in the reversion is inversely proportional to the density of the mobile monopoles. This implies that in the region of light monopoles, impurities do not favor the formation of extended Dirac chains. In this region, the samples with few impurities show a rich dynamic in very extensive Dirac chains and two distinct reversion phases. In the first phase, monopoles appear, generally at the ends and moving to the opposite extremes. These first monopoles disappear, producing a first plateau in the hysteresis curve. Then for a magnetic value close to coercitivity, new 
monopoles and the corresponding Dirac chains are produced. The second chains are shorter than the first. A second plateau is produced and then the samples reach total magnetization. This behavior can be observed in Figure 5, which shows the magnetization for a simulation with the same parameters as shown in Figure 4, but in the limit case where there are no impurities $(s=0)$. The same figure shows the heavy monopole regime without impurities. We can note that when the system reaches the coercitivity value, it remains in an extended plateau. During this plateau, the density of the mobile monopoles is at a maximum. There is then a second abrupt rise and a second plateau. In the final part of the magnetic reversion there is a third abrupt rise, reaching the maximum value of magnetization. It is only in the last rise that the density of defects goes from a low to a maximum value.

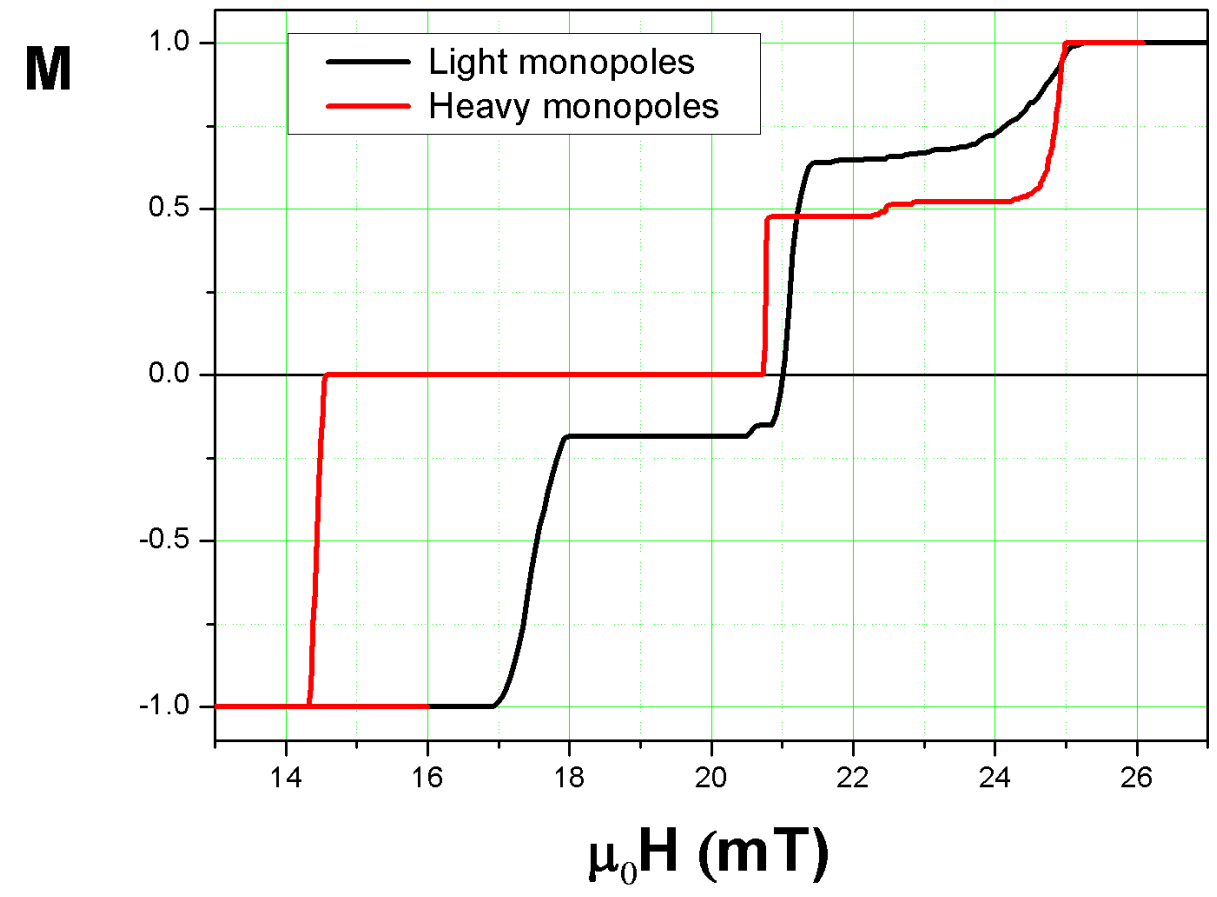

Figure 5. Magnetization for a simulation with the same parameters as shown in Figure 4, but in the limit case where there are no impurities $(s=0)$.

\section{Conclusion}

Magnetic reversion in artificial spin ice systems with hexagonal geometry presents two different dynamic mechanisms of elemental excitations, depending on the magnetic proper- 
ties of the individual nanoislands that form the array. In one case we can appreciate that reversion occurs with the appearance of a large number of monopoles that however remain static in the sample. In the other case, we can note the low number of monopoles that move great distances in the samples with very extensive Dirac chains. Impurities in the sample condition the number of these heavy and light monopoles. In the heavy monopole regime, the impurities decrease the number of monopoles and in the light monopole regime the opposite occurs, increasing the number of monopoles.

\section{Acknowledgments}

The author acknowledge the financial support of FONDECYT program grant 11100045.

[1] Wang, R. F. et al. Artificial "spin ice" in a geometrically frustrated lattice of nanoscale ferromagnetic islands. Nature 439, 303-306 (2006).

[2] Mengotti, E. et al. Building Blocks of an artificial kagome spin ice: Photoemission electron microscopy of arrays of ferromagnetic islands. Phys. Rev. B 78, 144402 (2008).

[3] Remhof, A. et al. Magnetostatic interactions on a square lattice. Phys. Rev. B 77, 134409 (2008).

[4] Ladak, S., Read D. E., Branford W. and Cohen L. F., New J. Phys. 13063032 (2011).

[5] Mellado P. Petrova O, Shen Y. C. and Tchernyshyov O., Phys. Rev. Lett. 105187206 (2010).

[6] Ladak S., Read D, Tyliszczak, Branford W. R. and Cohen L. F., New J. Phys. 13, 023023 (2011).

[7] Ladak S, Read D, Perkins G. K., Cohen L. F. and Branford W. R., Nature Phys. 6, 359 (2010).

[8] Mengotti, E. et al. Real-space observation of emergent magnetic monopoles and associated Dirac strings in artificia kagome spin ice. Nature phys. 7, 68-74 (2011).

[9] Morgan J. P., Stein A., Langridge S. and Marrows C. H., New Journal of Physics 13 (2011) 105002.

[10] Dirac, P. A. M. Quantised singularities in the electromagnetic field. Proc. R. Soc. Lond. A 133, 60-72 (1031). 
[11] Phatak C, Petford-Long A K, Heinonen O, Tanase M and De graef M 2011, Phys. Rev. B 83 174431.

[12] Budrikis Z, Politi P and Stamps R L 2010 Phys. Rev. Lett. 105017201.

[13] León A, arXiv:submit/0534000 [ cond-mat.mes-hall] 16 Aug 2012, submit New Journal of Physics (2012).

[14] León A, Comp. Phys. Com. (2012), 183, 10.

[15] León A, Phys. Lett. A 375 (2011) 4190-4197. 